\title{
A Survey on Optimization Algorithms for Optimizing the Numerical Functions
}

\author{
Ginnu George \\ Computer Science and Engineering Department \\ Karunya University
}

\author{
Kumudha Raimond, PhD. \\ Computer Science and Engineering Department \\ Karunya University
}

\begin{abstract}
Till now many optimization algorithms are available for solving complex problems. The algorithm that has created an impact is the numerical function optimization. Some of the existing optimization algorithms are classified into evolutionary computing and metaheuristic methods. A comprehensive survey on numerical function optimization is presented below with a deeper view into different aspects of the algorithms.
\end{abstract}

Keywords: Evolutionary Computing, Metaheuristic methods.

\section{INTRODUCTION}

Computational Intelligence (CI) is one of the popular approaches of Artificial Intelligence (AI). CI is mainly used to optimize the complex problems. Optimization is used to find the best solution out of the solution space. Numerical function optimization plays an important role in optimizing the objective function. Some of the optimization algorithms used for optimizing the numerical functions is categorized into evolutionary computing and metaheuristic methods. Swarm Intelligence (SI), Genetic Algorithm (GA) comes under evolutionary computing and Simulated Annealing (SA), Tabu Search (TS) and Hill Climbing comes under metaheuristic methods. Swarm Intelligence includes Bee(s) Algorithms (BA), Artificial Bee Colony algorithm (ABC), Particle Swarm Optimization (PSO), and Ant Colony Optimization (ACO). $\mathrm{ABC}$ is one of the popular swarm intelligent approaches which are used for optimizing the numerical functions. Some of the optimization algorithms are shown below in fig.1.

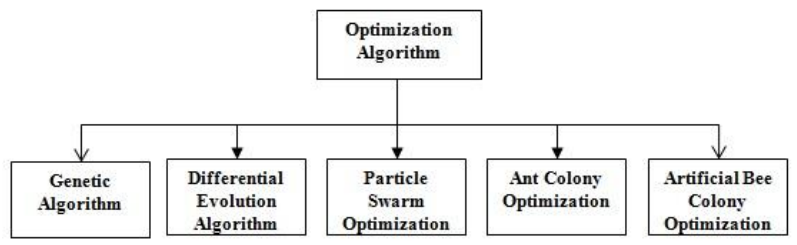

Fig. 1 Optimization Algorithms

PSO is an optimization technique based on the social behavior of group of organization. The main goal of PSO is that it mainly accelerates towards the P-best and G-best locations. ACO is a good optimization algorithm which mimics the searching behavior of ants. As soon as the ants receive their food source it immediately checks the quality of the food source and then it stores some of the food source in their hive.
During their return trip, it releases the pheromone hormone to guide their search. ABC is introduced by Dervis Karaboga in the year 2005 , which is mainly motivated by the searching behavior of bees. $\mathrm{ABC}$ is also known as population-based search procedure. Also, the original $\mathrm{ABC}$ algorithm combines both local search methods and global search methods that are being performed by the employed and onlooker bees for balancing the exploration and exploitation capacity. GA constitutes the natural selection and genetic recombination. GA chooses the solutions randomly from the population and then applies the genetic operators such as mutation and crossover for creating the new offspring. GA uses the historical information for investigating on new search areas in order to improve the performance. The main advantage of GA is that it performs global search. BA is an optimization technique which is inspired by the searching behaviour of bees to find the best nearly optimal solution. SA is mainly used with the GA where mutation operator is used. SA starts with the high rate of mutation operator and probably decreases the rate. TS performs same as SA where SA produces only a single mutated solution but TS produces many mutated solutions and selects the one which is having the lowest energy. Hill Climbing is a mathematical technique which is mainly for local search. The whole optimization algorithms perform until the termination criterion satisfies.

\section{SWARM INTELLIGENCE}

SI can be defined as the searching behavior of intelligent swarms. SI mainly comes under evolutionary computing. SI includes $\mathrm{ABC}, \mathrm{BA}, \mathrm{PSO}$ and $\mathrm{ACO}$.

\subsection{ABC ALGORITHMS}

D.Karaboga.et.al [1] in 2008 compares the performance of $\mathrm{ABC}$ algorithm with Differential Evolution (DE), PSO and Evolutionary Algorithm (EA). The ABC algorithm analyzes the different control parameter values. ABC algorithm is performed for five benchmark functions. The results show that $\mathrm{ABC}$ algorithm performs better with respect to the other algorithms that are mentioned above and also $\mathrm{ABC}$ is used for solving the complex problems. W. D. Karaboga.et.al [4] explains about the comparative study of the $\mathrm{ABC}$ Algorithm. In [4], the result that is produced by the $\mathrm{ABC}$ algorithm has been compared to the other optimization algorithms such as PSO, GA, DE and Evolution Strategies (ES). PSO is an optimization technique based on the social behavior of group 
of organization. The main goal of PSO is that it mainly accelerates towards the P-best and G-best locations.

GA constitutes the natural selection and genetic recombination. GA chooses the solutions randomly from the population and applies the genetic operators such as mutation and crossover for creating the new offspring. Performing global search is the main strength of the GA algorithm. DE works similar to GA algorithm. The main difference between $\mathrm{DE}$ with that of GA algorithm is that in GA crossover operator has been used whereas in DE, mutation operator is used. In $\mathrm{ABC}$, two types of mechanisms are introduced in order to control the diversity of the population and they are described as: (i) the randomly chosen part of the parent has been modified for obtaining the trial solution (ii) other than changing the part of the solution that is randomly chosen, the complete solution is being removed and then a new solution is inserted with the help of the scout. The two mechanisms that are described above helps to enhance the global search ability and the premature convergence problem are also getting prevented. Thus, due to the above reasons ABC shows much better results with the multimodal problems and can be concluded that the performance of $\mathrm{ABC}$ is higher than that GA, DE, PSO and ES. S. Pan.et.al [5] introduces an enhanced $\mathrm{ABC}$ known as Interactive $\mathrm{ABC}$ (I-ABC) algorithm. I-ABC mainly considers the universal gravitation between the bees in order to overcome the limitations of $\mathrm{ABC}$ algorithm. To know the better performance of I-ABC, the I-ABC is compared with that of PSO and ABC and also I-ABC, PSO and ABC has been applied to five benchmark functions. The results show that the I-ABC is very best in finding the better solution when compared to that of $\mathrm{ABC}$ and PSO with the help of the universal gravitation which is introduced to the onlooker bees. Also, I-ABC performs well in solving the complex combinatorial problems. . R. Akbari.et.al [6] presents a novel Bee Swarm Optimization (BSO) algorithm which is a population based search technique with the help of the honey bees. BSO presents two extensions for improving the performance.BSO algorithm introduces repulsion factor and penalizing fitness (RP) as the first extension for preventing from the stagnation problem and also time-varying weights (TVW) is introduced for balancing exploration and exploitation capacity. BSO and its two extensions have been compared with the $\mathrm{ABC}$ and also BSO is applied to six benchmark functions. Results show that the BSO with its two extensions performs best with respect to $\mathrm{ABC}$ algorithm. W.zou.et.al [7] introduces Cooperative $\mathrm{ABC}$ (CABC) for solving data clustering problems. Data clustering can be defined as the grouping of data into different clusters. The main goal of data clustering is that to make the data in a single cluster which requires high similarity features. K-means clustering algorithm is used in the $\mathrm{CABC}$ algorithm. $\mathrm{CABC}$ is used for solving six benchmark functions and the results produced by the $\mathrm{CABC}$ have been compared with $\mathrm{ABC}$, PSO and CPSO. The results show that the CABC outperforms than other algorithms mentioned above. G. zhu.et.al [8] proposes Gbest-guided ABC (GABC) algorithm by keeping the idea of the global best solution into the search equation for improving the exploitation capacity. GABC has been compared with $\mathrm{ABC}$ algorithm and the proposed algorithm is applied on six numerical benchmark functions. Results show that the GABC algorithm performs well compared to that of $\mathrm{ABC}$ algorithm R.Venkata Rao.et.al [13] proposes the optimization aspects of a multi-pass milling operation. The minimization of the production time is the main objective of [13]. For determining the process parameters cutting strategies are proposed. Feed, the depth of cut in each pass, number of passes and the cutting speed are the various cutting strategies. Three non tradition optimization algorithms such as ABC, PSO and SA are used for performing the optimization technique. The experimental results show that $\mathrm{ABC}$ and $\mathrm{PSO}$ are having better performance when compared to $\mathrm{SA}$ in terms convergence iteration. D.Karaboga.et.al [14] proposed a modified ABC algorithm for solving the constrained problems. [14] Uses Deb's rules for the constraint handling which consists of three simple rules and a probabilistic selection scheme. The modified $\mathrm{ABC}$ algorithm has been compared with that of state- of- the- art algorithms and analyses the statistical parameter. Hence, the modified ABC algorithm results with a better performance and efficiently solves the constrained optimization problems. T. Achalakul.et.al [15] presents the best-so-far selection in ABC. In the proposed method the best solution that is obtained are shared globally among the entire population. The proposed method is carried out into two sets of problems such as benchmark problems and image registration applications. The experimental results show that the above proposed method produces better quality solutions and faster convergence than the $\mathrm{ABC}$ algorithm. C. Ozturk.et.al [16] presents a novel clustering approach using $\mathrm{ABC}$ algorithm. Clustering is mainly used to identify the homogeneous group of data. K-means algorithm is used. The proposed $\mathrm{ABC}$ algorithm is carried out on different benchmark functions. The results that are produced are compared with that of PSO algorithm and nine classification techniques. In order to demonstrate the results, thirteen typical data sets from the UCI Machine Learning Repository are used. Thus, the results show that the ABC is very efficient in solving the data clustering problems. S.Liu.et.al [17] proposes an Improved ABC (IABC) algorithm for global optimization. The IABC proposes improved search equations such as $\mathrm{ABC} /$ best $/ 1$ and $\mathrm{ABC} / \mathrm{rand} / 1$. Also, this method describes the parameter $M$ which is used for controlling the elements between the candidate source position and the old one. The chaotic systems and the opposition-based learning method are employed during the generation of initial population for enhancing the global convergence speed. The proposed method is carried out on different benchmark functions and the experimental results show that the $\mathrm{ABC}$ is having good performance in solving the complex optimization problems. R. Akbari [18] investigates three bee algorithms for solving Resource Constrained Project Scheduling Problem (RCPSP). $\mathrm{BA}, \mathrm{ABC}$ and $\mathrm{BSO}$ are the three bee algorithms used in RCPSP. For solving RCPSP, three phases are proposed such as initialization, update and termination. A set of schedules are generated during the first phase. Each algorithm is described in the second phase and in third phase each 
algorithm is repeated until the termination condition is satisfied. In addition to the proposed methods, a constraint handling method and three local search methods are also introduced for achieving good efficiency. The experimental results are compared with the state-of-art algorithms and also produce better results in solving RCPSP problems. G.S.
M.Sonmez.et.al [21] describes $\mathrm{ABC}$ algorithm for optimizing the Truss structures. The main objective of the $\mathrm{ABC}$ algorithm is to minimize the weight of the truss. An adaptive penalty function is used with the $\mathrm{ABC}$ algorithm for improving the efficiency.

Table 1. ABC Algorithms

\begin{tabular}{|c|c|c|c|c|c|c|}
\hline $\begin{array}{c}\text { S.N } \\
\mathbf{0}\end{array}$ & Author & Algorithm used & BMF & Metrics & $\begin{array}{l}\text { Compared with } \\
\text { Algorithms }\end{array}$ & Remarks \\
\hline 1 & $\begin{array}{l}\text { P.Tsai et.al., } \\
2009 \text { [5] }\end{array}$ & $\begin{array}{l}\text { Interactive } \\
\quad \mathrm{ABC}\end{array}$ & 5 & $\begin{array}{l}\text { Iterations, Fitness } \\
\text { value }\end{array}$ & $\mathrm{ABC}$ and $\mathrm{PSO}$ & $\begin{array}{l}\text { I-ABC perform better in } \\
\text { terms of time and } \\
\text { fitness value }\end{array}$ \\
\hline 2 & $\begin{array}{l}\text { G. Zhu et.al., } \\
2010[8]\end{array}$ & $\begin{array}{c}\text { Gbest-guided } \\
\text { ABC }\end{array}$ & 6 & $\begin{array}{l}\text { Iterations, Mean } \\
\text { of best function } \\
\text { value }\end{array}$ & $\mathrm{ABC}$ & $\begin{array}{lr}\text { GABC with } & \mathrm{C}=1.5 \\
\text { outperforms } & \mathrm{ABC} \\
\text { Algorithm } & \end{array}$ \\
\hline 3 & $\begin{array}{l}\text { D.Karboga } \\
\text { et.al.,2011 [14] }\end{array}$ & $\begin{array}{l}\text { Modified ABC } \\
\text { algorithm. }\end{array}$ & 13 & $\begin{array}{l}\text { Iterations, global } \\
\text { optimum value }\end{array}$ & $\begin{array}{l}\text { State-of-art } \\
\text { algorithms. }\end{array}$ & $\begin{array}{l}\text { Cannot find optimum } \\
\text { solutions for some } \\
\text { problems }\end{array}$ \\
\hline 4 & $\begin{array}{l}\text { T. Achalak } \\
\text { et.al., } 2011 \text { [15] }\end{array}$ & Best-so-far $\mathrm{ABC}$ & 6 & Iteration, Mean & $\mathrm{ABC}$ algorithm & $\begin{array}{l}\text { Convergence rate is } \\
\text { high }\end{array}$ \\
\hline 5 & $\begin{array}{l}\text { M. Sonmez } \\
2011 \text { [21] }\end{array}$ & $\mathrm{ABC}-\mathrm{AP}$ & 6 & $\begin{array}{l}\text { Iterations, } \\
\text { Weights. }\end{array}$ & $\mathrm{ABC}$ algorithm & \begin{tabular}{lrr} 
Robust & \multicolumn{2}{c}{ performance } \\
with a $100 \%$ & success \\
rate. & & \\
\end{tabular} \\
\hline 6 & $\begin{array}{l}\text { B.Akay et.al., } \\
2012 \text { [23] }\end{array}$ & $\begin{array}{l}\text { Modified ABC } \\
\text { for real parameter } \\
\text { optimization }\end{array}$ & NIL & MR, Error rate. & $\begin{array}{l}\text { State-of-art } \\
\text { Algorithms }\end{array}$ & $\begin{array}{l}\text { Produces a promising } \\
\text { result on } \\
\text { functions. }\end{array}$ \\
\hline 7 & $\begin{array}{l}\text { A. Rajasekar } \\
\text { et.al., } \\
2012 \text { [24] } \\
\end{array}$ & $\begin{array}{c}\text { Hybrid } \\
\text { Differential ABC }\end{array}$ & 10 & Iterations, Mean & $\mathrm{ABC}$ and $\mathrm{DE}$ & $\begin{array}{l}\text { Performance is good } \\
\text { than } A B C \text { and DE. }\end{array}$ \\
\hline 8 & $\begin{array}{l}\text { Y.Zhu et.al., } \\
2012[25]\end{array}$ & Hybrid ABC & 10 & Mean, Time & $\mathrm{GA}, \mathrm{ABC}$ and $\mathrm{PSO}$ & $\begin{array}{l}\text { HABC uses more time } \\
\text { than PSO and GA but } \\
\text { its results are much } \\
\text { better }\end{array}$ \\
\hline
\end{tabular}

B.Akay.et.al [23] presents a modified ABC algorithm for realparameter optimization. Effects of the perturbation rate, Scaling Factor (SF) and limit parameter are investigated the real parameter optimization. Only one control parameter known as limit is present in the basic ABC. Hence, the convergence rate is poor. For improving the convergence rate,some modifications are introduced in basic $\mathrm{ABC}$ algorithm. A control parameter called Modification rate is proposed for strengthening the convergence rate. Another modification is with respect to the SF, an adaptive SF is introduced for improving the convergence and the exploitation capacity. Thus, the results show that the modified $\mathrm{ABC}$ is very efficient in solving the real-parameter optimization. A. Rajasekar.et.al [24] explains a Hybrid Differential ABC Algorithm (HDABC). Long computational time is the great disadvantage of many optimization algorithms. In order to overcome the above disadvantage a HDABC is proposed. HDABC combines ABC with DE. In HDABC, two hybridization schemes are proposed such as the staged pipelining type and the other is additional-operator type. HDABC first select $\mathrm{n}$ differential vectors and chooses 10 best food sources depending upon the fitness values and these values will be initial population of the DE algorithm which is tuned by the mutation, crossover and selection operators and finally brings the best value. The proposed HDABC is applied to some of the test functions and is compared to $\mathrm{DE}$ and $\mathrm{ABC}$ algorithm and the results show that the HDABC as better than $\mathrm{DE}$ and $\mathrm{ABC}$ algorithms. Y.Zhu.et.al [25] presents a new approach of data clustering using hybrid $\mathrm{ABC}$ algorithm. To overcome the disadvantages of the basic $\mathrm{ABC}$ algorithm crossover operator is introduced. The main goal of the crossover operator is to exchange the full information among the bees and to find the best one that is utilized. In order to determine the good performance, the HABC algorithm is executed on six datasets from the UCI machine learning database and it is applied to the clustering problems. The experimental results show that the HABC performs better. G.Li.et.al investigates over the $\mathrm{ABC}$ and proposed Improved $\mathrm{ABC}(\mathrm{I}-\mathrm{ABC})$ in order to overcome the limitations of $\mathrm{ABC}$. In I-ABC acceleration coefficients and inertia weight are introduced in search equation of the $\mathrm{ABC}$. The main strength of $\mathrm{I}-\mathrm{ABC}$ is having fast convergence speed than $\mathrm{ABC}$ and its main limitation is that in some cases I-ABC will get trapped in local optimal solutions. In order to prevent the above limitation Prediction Selection ABC (PSABC) is introduced. Prediction and Selection are the main strengths of $A B C$. Three solution Search equations are introduced in PS-ABC to overcome the limitation of I-ABC. The search equations from $\mathrm{ABC}, \mathrm{I}-\mathrm{ABC}$ and $\mathrm{GABC}$ are the solution search equations defined in the search equation of PS-ABC. The experimental results produced with the help of three search equations in PS$\mathrm{ABC}$ are compared with that of $\mathrm{ABC}$ and $\mathrm{I}-\mathrm{ABC}$ and it is 
applied to that of 13 benchmark functions. The results show that PSABC is having fast convergence speed and will not get trapped into the local optimal solutions and thus proves that PSABC is better to that of $\mathrm{ABC}$ and IABC. The different ABCalgorithms are explained in Fig 2.

\subsection{BA ALGORITHMS}

In [9], the original BA has been modified in order to solve GAP. The first modification is that using EC-length parameter instead of ngh parameter. Second modification is with respect to scout bees. For GAP, an adaptive penalty coefficient is introduced in which the re-initialization mechanism is employed whenever the solution did not get improved. Two types of ejection chain mechanisms are used such as shift and double shift mechanisms. The above two mechanism is employed to each employee bee which performs local search and finally obtains nearly optimal solutions. The modified GAP is applied on some GAP problems and the results show that the modified BA is efficient in solving GAP problems. Das.et.al [19] introduces a hybrid bee(s) algorithm to solve Container Loading (CL) problems. The main objective of CL problems is to load a set of boxes into a single large container for maximizing total volume of the packed items. This method uses heuristic filling procedure. Different operators like 1-flip, swap and k-flip operators are used in CL problems.

Table 2. Bee(s) Algorithms

\begin{tabular}{|c|c|c|c|c|c|c|}
\hline $\begin{array}{l}\text { S. } \\
\text { no }\end{array}$ & Author & Algorithm used & BMF & Metrics & $\begin{array}{l}\text { Compared } \\
\text { with } \\
\text { Algorithms }\end{array}$ & Remarks \\
\hline 1 & $\begin{array}{l}\text { L.Ozbak } \\
\text { et al.,2010 [9] }\end{array}$ & Bees algorithm & NIL & $\begin{array}{l}\text { Mean, } \\
\text { Time }\end{array}$ & NIL & $\begin{array}{l}\text { Performance is good for } \\
\text { solving GAP but no parameter } \\
\text { optimization is carried out. }\end{array}$ \\
\hline 2 & $\begin{array}{l}\text { G.S. Das. } \\
\text { et al., } 2011 \text { [19] }\end{array}$ & $\begin{array}{l}\text { Hybrid Bee(s) } \\
\text { Algorithm(Heuri } \\
\text { stic filling } \\
\text { procedure) }\end{array}$ & $\begin{array}{l}\text { NIL } \\
2 \text { test problem } \\
(\mathrm{CL})\end{array}$ & $\begin{array}{l}\text { NIL, } \\
\text { Utilization } \\
\text { rate. }\end{array}$ & NIL & $\begin{array}{l}\text { Capable of solving NP-hard } \\
\text { optimization problems. }\end{array}$ \\
\hline 3 & $\begin{array}{l}\text { M.Rahimi } \\
\text { et al., } 2011 \text { [20] }\end{array}$ & $\begin{array}{l}\text { Genetic Bee K- } \\
\text { Means } \\
\text { Algorithm } \\
\text { (GBTKC) }\end{array}$ & $\begin{array}{l}3 \text { well known } \\
\text { test problem. }\end{array}$ & NI, Mean & $\begin{array}{l}\text { HBA, GA, } \\
\text { TS } \\
\text { algorithm. }\end{array}$ & $\begin{array}{ll}\text { GBTKC shows better } \\
\text { performance but having long } \\
\text { CPU time. }\end{array}$ \\
\hline 4 & $\begin{array}{l}\text { M.Yuan Cheng. } \\
\text { et al., } 2012[22]\end{array}$ & Particle BA & CSL & $\begin{array}{l}\text { NI, Fitness } \\
\text { value. }\end{array}$ & $\begin{array}{l}\text { BA and } \\
\text { PSO. }\end{array}$ & $\begin{array}{l}\text { Fitness and mean values for } \\
\text { PBA are better }\end{array}$ \\
\hline
\end{tabular}

M.Rahimi.et.al [20] presents a hybrid algorithm for data clustering using honey bee algorithm, genetic algorithm and k-means method. On the basis of Honey Bee Algorithm (HBA) a Genetic Bee Tabu K-Means Clustering Algorithm (GBTKC) is introduced for improving the efficiency. Also, Kmeans algorithm is proposed for seeking the global optimum. GBTKC is executed on some of the data sets and the results produced show that the method proposed is very efficient in converging optimal solutions. M.Yuan Cheng.et.al presents the Construction Site Layout (CSL) [22] design. The main goal of CSL problems is to identify the most feasible location for a set of interrelated objects that maximizes the design by minimizing the total cost. The Particle Bee Algorithm (PBA) is introduced for solving CSL problems. PBA algorithm mainly compares the performance of both BA and PSO algorithms. A Neighbourhood Window (NW) technique and Self-Parameter-Updating (SPU) are proposed in order to improve the search efficiency of PBA and to prevent from the trapping into local optimal solutions. The performance of PBA is compared to BA and PSO and the experimental results show that the PBA algorithm is efficient in solving the CSL problems than the above mentioned algorithms. Fig.2 explains the different bee(s) algorithms.

\subsection{PSO ALGORITHMS}

Tsoulos.et.al [11] presents Enhancing PSO methods. PSO is used mainly for global optimization. In order to increase the speed and efficiency three modifications of basic PSO method has been proposed. New stopping rule, similarity check and application of local search method are the three proposed modifications. Enhancing PSO methods have applied on three PSO variants and other test functions and results show that there is better performance in terms of speed and efficiency.H. Modares.et.al [12] proposes the parameter identification problem for chaotic dynamic systems. A novel computation technique known as Improved PSO (IPSO) is proposed to solve the parameter identification problem. The parameter accuracy and computational time of proposed IPSO has been compared with that of GA and Standard PSO (SPSO). IPSO is mainly used to measure the variation of parameters. In IPSO, a sentry particle is proposed in order to determine the changes in the parameters. The experimental results show that the proposed IPSO is better than GA and SPSO in finding the optimal parameters.

\subsection{ACO ALGORITHMS}

J.Yang.et.al [10] proposes an Improved Ant Colony Optimization (I-ACO) algorithm. The proposed algorithm is used to solve the mobile agent routing problem. In I-ACO algorithm, genetic algorithm that is mutation operator is introduced and also modifies the global updating rules. I-ACO has been compared with that of GA, SA and basic ACO. The experimental results show that the I-ACO escapes from the local optimal solutions and also having faster convergence speed. With the help of the I-ACO algorithm, the fitness and the perfectness of the whole system has been enhanced. 
Table 3. Other Optimization Algorithms

\begin{tabular}{|c|l|c|c|c|c|c|}
\hline S.No & \multicolumn{1}{|c|}{ Author } & $\begin{array}{c}\text { Algorithm } \\
\text { used }\end{array}$ & BMF & Metrics & $\begin{array}{c}\text { Compared with } \\
\text { Algorithms }\end{array}$ & Remarks \\
\hline 1 & W.Zhao.et al., & Novel EA & 24 & $\begin{array}{c}\text { Iterations, best } \\
\text { value }\end{array}$ & FEP and LEP & $\begin{array}{c}\text { Convergence rate is } \\
\text { fast. }\end{array}$ \\
\hline 2 & E.Rashdi.et al., & GSA & 23 & $\begin{array}{l}\text { Iterations, Average } \\
\text { best value }\end{array}$ & CFO and PSO & $\begin{array}{c}\text { GSA provides } \\
\text { superior results }\end{array}$ \\
\hline 3 & $\begin{array}{l}\text { I.G.Tsoulos.et } \\
\text { al., 2010 [11] }\end{array}$ & $\begin{array}{l}\text { Enhanced PSO } \\
\text { Methods }\end{array}$ & NIL & Iterations, variance & NIL & $\begin{array}{c}\text { Enhance speed and } \\
\text { efficiency. }\end{array}$ \\
\hline
\end{tabular}

\section{OTHER OPTIMIZATION ALGORITHMS}

Zhao.et.al [2] introduces a novel evolutionary algorithm (NEA) for solving the global numerical optimization problems. In NEA, the initial population is produced by both deterministic and the random ones. In order to make NEA better, a decent scale function and a mutation operator is being introduced. The decent scale function is employed with crossover operator to find best solution quickly whereas the mutation operator is introduced for increasing the convergence rate. NEA algorithm executes over 24 benchmark functions and the results show that the convergence rate is higher than that of EA and GA. E.Rashedi.et.al [3] presents a gravitational search algorithm (GSA) on the gravitational laws.GSA consists of searcher agents who interacts with each other with the help of newton's gravity and the laws of motion. Higher gravitational mass constitutes higher performance. Hence, in GSA all agents are attracted towards greater gravitational mass for achieving higher performance. The GSA algorithm has been compared with CFO and PSO and the results show that the GSA is having higher performance than that of CFO and PSO.

\section{GENETIC ALGORITHMS}

Dongsheng Liu [29] presents an Improved GA (I-GA) based on SA and Quantum Computing Strategy. The I-GA is mainly used for mining association rules. The results show that by using I-GA more association rules can be generated. D.Alkamin et.al [30] presents a hybrid GA which is mainly applied on clustering problems. Hybrid GA performs local search and global search and prevents from getting trapped of the local optimal solutions. The experiment is conducted on real data sets and results show that hybrid GA outperforms than the other clustering algorithms.

\section{OTHER METAHEURISTIC METHODS}

Li.Wang et.al [27] presents an SA approach along with the hypothesis test (HT) for stochastic optimization.HT is mainly used to jump from getting trapped into local optimal solutions and also it is used to estimate the performance of the solution. M.Sebag et.al [28] presents an Hill Climber approach used for optimizing the functions in binary search spaces. The main goal of Hill Climber is to speed up the search. The experiment is investigated on 900 bit problems and results show that it outperforms than genetic algorithm and Evolution strategies.

\section{CONCLUSION}

The survey has been investigated over some of the optimization algorithm such as ABC, PSO, ACO, BSO, GSA etc. The survey shows that when compared to all other optimization algorithms PS-ABC produces a better result due to the prevention of the trapping from the local optimal solutions. The main difference of PS-ABC when compared to the other optimization algorithms is that in PS-ABC three search equations are used and the corresponded fitness values are calculated whereas in other optimization algorithms they just modify the search equation of the original $A B C$ algorithm. Hence, PS-ABC shows better performance with respect to the numerical function optimization and the mean values.

\section{REFERENCES}

[1] D. Karaboga, B. Basturk "On the performance of artificial bee colony algorithm", Applied Soft Computing, vol. 8, pp. 687-697, June.2008.

[2] W. Zhao, W. Wang, Y. Wang "A novel evolutionary algorithm for global numerical optimization with continuous variables", Applied Soft Computing, vol. 18, pp. 345-351, Nov.2008.

[3] E. Rashedi, H. Nezamabadi-pour, S. Saryazdi "GSA: a gravitational search algorithm", Information Sciences, vol. 179, pp. 2232-2248, Mar.2009.

[4] D. Karaboga, B. Akay "A comparative study of Artificial Bee Colony algorithm", Applied Mathematics and Computation, vol.14, pp. 108-132, Sept. 2009.

[5] P.Tsai, J.S. Pan.et.al "Enhanced Artificial Bee Colony Optimization”, Innovative Computing, vol.5, pp. 13494198, Aug.2009.

[6] R. Akbari, A. Mohammadi, K. Ziarati “A novel bee swarm optimization algorithm for numerical function optimization", Communications in Nonlinear Science and Number Simulation, vol. 15, pp. 3142-3155,Nov. 2010 .

[7] W. Zou, Y. Zhu, H. Chen and X . Sui "A Clustering Approach Using Cooperative Artificial Bee Colony 
Algorithm", Discrete Dynamics in Nature and Society, vol.10, pp. 1-16, Oct.2010.

[8] G. Zhu, S. Kwong "Gbest-guided artificial bee colony algorithm for numerical function optimization", Applied Mathematics and Computation, vol. 217, pp. 3166-3173, Sept. 2010.

[9] L. Ozbakir, A. Baykasoglu, P. Tapkan " Bees algorithm for generalized assignment Problem", Applied Mathematics and Computation, vol. 215 , pp. 37823795,Dec. 2010

[10] J.G. Yang, Y.B. Zhuang "An improved ant colony optimization algorithm for solving a complex combinatorial optimization problem", Applied Soft Computing, vol.10, pp.653-660, Oct.2010.

[11] I . G. Tsoulos, A. Stavrakoudis "Enhancing PSO methods for global optimization", Applied Mathematics and Computation,vol. 216 , pp. 2988-3001, May.2010.

[12] H. Modares, A. Alfi, M.-M. Fateh "Parameter identification of chaotic dynamic systems through an improved particle swarm optimization", Expert Systems with Applications, vol. 37, pp. 3714-3720, Aug.2010.

[13] R. Venkata Rao, P.J. Pawar, "Parameter optimization of a multi-pass milling process using non-traditional optimization algorithms", Applied Soft Computing, vol.10, pp.445-456,Aug. 2010.

[14] D. Karaboga, B. Akay "A modified Artificial Bee Colony (ABC) algorithm for constrained optimization problems", Applied Soft Computing, vol.11, pp.30213031, Dec.2011.

[15] A.Banharnsakun, T. Achalakul, B. Sirinaovakul "The best-so-far selection in Artificial Bee Colony algorithm", Applied Soft Computing, Vol.11, pp. 2888-2901, Dec.2011.

[16] D. Karaboga, C. Ozturk "A novel clustering approach: Artificial Bee Colony (ABC) Algorithm", Applied Soft Computing, vol. 11, pp. 652-657, Dec.2011.

[17] W. Gao, S. Liu "Improved artificial bee colony algorithm for global optimization", Information Processing, Vol.111, pp. 871-882, Jun.2011.

[18] K. Ziarati, R. Akbari, V. Zeighami "On the performance of bee algorithms for resource-constrained project scheduling problem", Applied Soft Computing, vol.11, pp. 3720-3733, Mar.2011.

[19] T. Dereli, G.S. Das, "A hybrid bee(s) algorithm for solving container loading Problems", Applied Soft Computing, vol.11, pp. 2854-2862, Dec.2011.

[20] M.AliShafia, M.Rahimi, R.Tavakolian "A Hybrid Algorithm for Data Clustering Using Honey Bee Algorithm,Genetic Algorithm and K-Means Method", Computer Science and Technology Research, vol.1, pp. 110-125, Dec.2011.
[21] M. Sonmez,"Artificial Bee Colony algorithm for optimization of truss structures", Applied Soft Computing, vol.11, pp. 2406-2418, May.2011.

[22] L.Chuan Lien, M.Yuan Cheng, "A hybrid swarm intelligence based particle-bee algorithm for construction site layout optimization", Expert Systems with Applications, vol.39, pp. 9642-9650, Dec.2012.

[23] B.Akay, D. Karaboga "A modified Artificial Bee Colony algorithm for real parameter Optimization", Information Sciences,vol.192, pp. 120-142, July.2012.

[24] Ajith Abraham, Ravi Kumar Jatoth, A. Rajasekhar "Hybrid Differential Artificial Bee Colony Algorithm", Computational and Theoretical Nanoscience, vol. 9, pp.1-9,Dec. 2012.

[25] X. Yan, Y.Zhu, W.G.Zou, L.Wang "A new approach for data clustering using hybrid artificial bee colony algorithm", Neurocomputing, vol.11, pp.1-10, Apr.2012.

[26] G. Li, P. Niu, X. Xiao, "Development and Investigation of Efficient Artificial Bee Colony Algorithm for Numerical Function Optimization," Applied Soft Computing, vol.12, pp. 320-332, Aug.2011.

[27] L.Wang and L.Zhang, "Stochastic Optimization using Simulated Annealing with hypothesis test,"Applied Mathematics and computation, vol.174, pp.1329-1342, Jun.2006.

[28] M. Sebag and M. Schoenauer, "A Society of Hill Climbers," Applied Soft Computing, vol.9, pp. 16,Sep.2009.

[29] D. Liu, "Improved Genetic Algorithm Based on Simulated Annealing and Quantum Computing Strategy," vol.11, pp. 1243-1249, Nov.2010.

[30] D.Alkamin and F.Varejao,"Hybrid Genetic Algorithm Applied to the Clustering Problem," vol.33, pp. 141-151, Jan.2012.

\section{AUTHOR'S PROFILE}

Ginnu George received her B.Tech from Sri Subramanya college of Engineering and Technology, affiliated to Anna University and currently doing M.tech in Karunya University.

Dr. Kumudha Raimond received her B.E from Arulmigu Meenakshi Amman College of Engineering, affiliated to Madras University and M.E from Government College of Technology, Coimbatore and Doctoral degree from Indian Institute of Technology, Madras, India. Her area of expertise is in intelligent systems. She is a Senior Member of International Association of Computer Science and Information Technology (IACSIT) and Member of Machine Intelligence Research Lab: Scientific Network for Innovation and Research Excellence. 